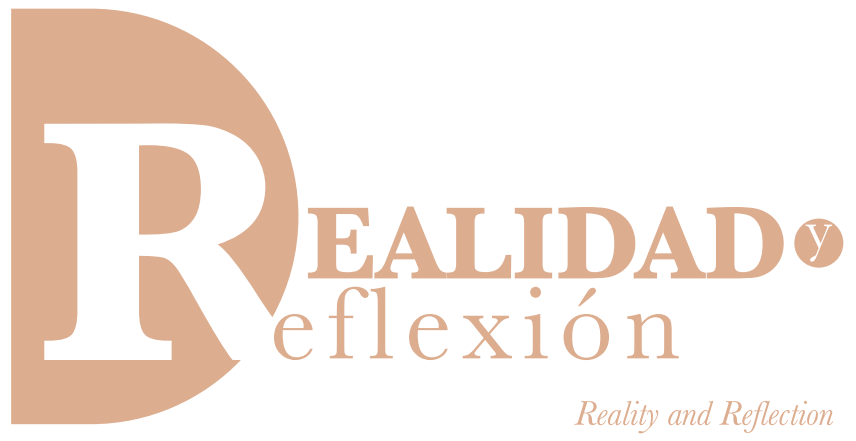

ISSN 1992-6510

e-ISSN 2520-9299

Año 17, N 46, San Salvador, El Salvador, Centroamérica. Revista Semestral Julio-Diciembre 2017

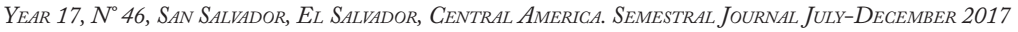

\title{
Redes sociales como componentes educativos en los entornos digitales de aprendizaje
}

\author{
Social networking as learning components in the digital \\ educational enviroments
}

\author{
Herberth Alexander Oliva ${ }^{1}$ \\ Especialista en Pedagogía, egresado de la Universidad Pedagógica de El Salvador. \\ Cursos de especialización en Southern Texas University, Instituto Católico de Barcelona, \\ Universidad de Tel Aviv y Universidad del Valle de Guatemala. \\ Investigador del Instituto de Ciencia, Tecnología e Innovación de la Universidad Francisco Gavidia (ICTI-UFG) \\ holiva@ufg.edu.sv \\ Recibido: 28 de agosto de 2017 \\ Aprobado: 12 de diciembre de 2017 \\ pág. $34-53$ \\ DOI: http://dx.doi.org/10.5377/ryr.v0i46.5506
}

\section{RESUMEN}

El presente artículo es una intelección académica, en el cual se aborda el estudio de las redes sociales como objeto de estudio la construcción (elemento epistémico) y desarrollo (elemento pedagógico didáctico) de aprendizajes significativos en Entornos Digitales de Aprendizaje. Esto permitirá crear un marco de aproximación epistemológica en el cual las redes tecnológicas de comunicación puedan percibirse como una herramienta que permite el aprendizaje colaborativo e involucra espacios de intercambio de información que fomentan la cooperación y significatividad de las redes sociales en la formación académica de los estudiantes y su proceso educativo.

Palabras clave: Redes sociales, aprendizaje, entorno personal de aprendizaje, entorno personal de aprendizaje mediado por la institución.

\section{ABSTRACT}

The present article is an academic intellection, in which the study of social networks is studied as an object of study the construction (epistemic element) and development (didacticpedagogical element) of significant learning in Digital Learning Environments. This will allow creating a framework of epistemological approach in which technological communication networks can be perceived as a tool that allows collaborative learning and involves spaces for information exchange that foster cooperation and significance of social networks in the academic formation of students and its educational process.

Keywords: Social networks, learning, personal learning environment, personal learning environment mediated by the institution.

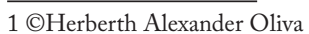




\section{Introducción}

La intención de este documento es poder consolidarse como un aporte bibliográfico en el cual se pueda plasmar las bondades de las redes sociales como herramienta para la enseñanza en la formación de estudiantes universitarios; de tal forma que se pueda derivar un marco de interpretación en el cual el conglomerado estudiantil pueda reconocer que tiene la necesidad de identificarse con otros miembros de iguales círculos sociales de convivencia. Esto hace que, en el contexto vertiginoso de la sociedad de la información y el conocimiento, las tendencias tecnológicas y acelerado ritmo de vida, dé pauta a la creación de herramientas y medios tecnológicos que faciliten la construcción de nuevos espacios de encuentro, a través de los cuales muchas personas se comunican con otros sin importar tiempos o latitudes con el único propósito de consolidar los nuevos tejidos sociales del posmodernismo educativo.

Las actuales dinámicas de interacción en los procesos de enseñanza y aprendizaje, permiten entender que las redes sociales que se presentan en internet, crean un marco de apertura en el cual se presenta la viabilidad de que un estudiante o un grupo de estos, pueda interactuar con sus similares mediante un acelerado intercambio en el que la misma dinámica educativa les permita poder crear espacios para coincidir en el pronunciamiento de problemas, ideas, aficiones, gustos etc. Todo ello mediante el uso de medios tecnológicos.

En la actualidad, los espacios de aprendizaje han hecho que las redes sociales le permitan adquirir a las generaciones nacidas en la era digital, potenciar una gran capacidad de aprendizaje usando tecnologías emergentes, las cuales se consolidan en una gran variedad de redes sociales como plataformas de comunicación y de almacenamiento de un gran caudal de conocimientos que son permanentemente actualizados.

Esto ha provocado que diversas instituciones enfocadas al análisis cuantitativo del uso del Internet como el Institute of Science for Internet Studies, calcule que en el 2017 sean aproximadamente 2 mil millones de usuarios de Internet quienes usan las redes sociales contando con un promedio de 1000 millones de cuentas registradas y con un promedio de 1.87 millones de usuarios activos mensuales (Birmingam, 2017).

Lo anterior permite comprender que usar las redes sociales en contextos educativos puede ser una alternativa con gran cantidad de posibilidades para mejorar las estrategias didácticas. A ello se le debe sumar el hecho de que son los mismos estudiantes quienes afirman tajantemente que combinar el uso de redes sociales, durante el desarrollo de sus clases o tareas, les permitió entender que podían mejorar sus competencias para el uso de la Web 2.0 y vieron cómo de forma significativa van dominando más recursos tecnológicos que les sirven como estrategia de enseñanza cuyo punto de inicio es la experiencia adquirida al emplearla como forma de aprendizaje.

El interés por realizar esta investigación surge de la observación de las tecnologías y cómo ellas se han empoderado cada vez más de las dinámicas al interior de los centros educativos, facilitando a los estudiantes una gran variedad de metodologías y principios didácticos 
sobre cómo aprender más sobre la base de la eficiencia y eficacia de la calidad educativa. s precisamente en ese contexto en donde autores como Frank Patterson quien es especialista en el uso de las redes sociales como herramientas formativas de Syracuse University argumenta que el uso de redes sociales en el desarrollo de procesos de aprendizaje, se ha convertido en un recurso tecnológico de gran aceptación y suma preferencia para los estudiantes; ya que consideran que el intercambio de documentos, imágenes, fotografías etcétera, les permite personalizar su enfoque educativo y canalizar en mejor forma el contenido de estudio, haciendo de la institución educativa un componente central en el cual los usos de las redes sociales deberán de ayudar a los educandos a promover debates, fomentar el análisis, y conocer información relevante para el desarrollo eficiente de las actividades formativas.

\section{Contexto global en el que se desarrollan redes sociales como componentes educativos en los entornos digitales de aprendizaje}

Los modos de vivir y relacionarse han cambiado con la irrupción tecnológica en diversos espacios de la vida social: la empresa, la escuela, la institución, la convivencia, el ocio, los medios de comunicación; están impregnados del uso de la tecnología, particularmente del internet. El uso de telefonía con opciones novedosas, las plataformas para uso pedagógico y las redes sociales están abriendo nuevos espacios y posibilidades de comunicación y aprendizaje. Castells (2001) en Coll (2008), se ha referido al Internet como un nuevo y complejo espacio global para la acción social y por extensión, para el aprendizaje y la acción educativa.
De acuerdo a Chan (s/a), la digitalización educativa comenzó a manifestarse en la década de los noventa, entonces, los sistemas educativos incluyeron entre sus materiales pedagógicos recursos multimedia, empujados por una cultura de la información digital y aunque las tendencias en el uso de las tecnologías se realizaron desde la digitalización, al correr de los años se ha caminado hacia la virtualización de los procesos educativos. Hoy en día existen planes de estudio 100\% virtuales en toda América Latina, en nivel medio superior, superior y posgrado que hacen del supuesto de Chan una realidad.

A pesar de que E1 Salvador está por debajo de los países de primer mundo en el uso de la tecnología en el ámbito educativo, una realidad es que nos encontramos en un contexto que pudiera invitar u obligar a la incursión y/o consolidación de los procesos pedagógico-didácticos a través del uso de herramientas tecnológicas. Para nadie es extraño el poder identificar que muchas agendas educativas de varios gobiernos en el continente americano buscan que se integren las TIC al proceso educativo como uno de los medios para mejorar la calidad de la educación.

En este sentido, Pérez (2012) habla de que "ya no puede entenderse los procesos de enseñanzaaprendizaje, donde los individuos se ponen en contacto con la información y el conocimiento disponible, sin la presencia poderosa y amigable de las Tecnologías de la Información y la Comunicación (TIC) y en particular de la red de redes, el internet".

Más allá del uso del internet como una herramienta para la comunicación y acceso a la información, se ha observado un fenómeno que ha cobrado interés para los investigadores educativos, 
el uso de las redes sociales en el ámbito educativo, a partir de la premisa de que "la vida cotidiana de las nuevas generaciones, sobre todo de los jóvenes, se configura mediatizada por las redes sociales virtuales, induciendo nuevos estilos de vida, de procesamiento de información, de intercambio, de expresión y de acción” (Pérez, 2012).

Muestra de ello es que al 2012, Facebook había superado los 800 millones de usuarios, Twitter los 200 millones, y Google+ registró 62 millones y la red española Tuenti alcanzó los 13 millones de usuarios.

Para el 2017, esta cifra se incrementó hasta llegar a los 1,900 millones de usuarios, lo cual ubica a la red social por encima de otras redes sociales populares como WhatsApp, Youtube, Instagram, entre otras (ver Gráfico n. ${ }^{\circ} 1$ ).

\section{Uso de redes sociales 2017}

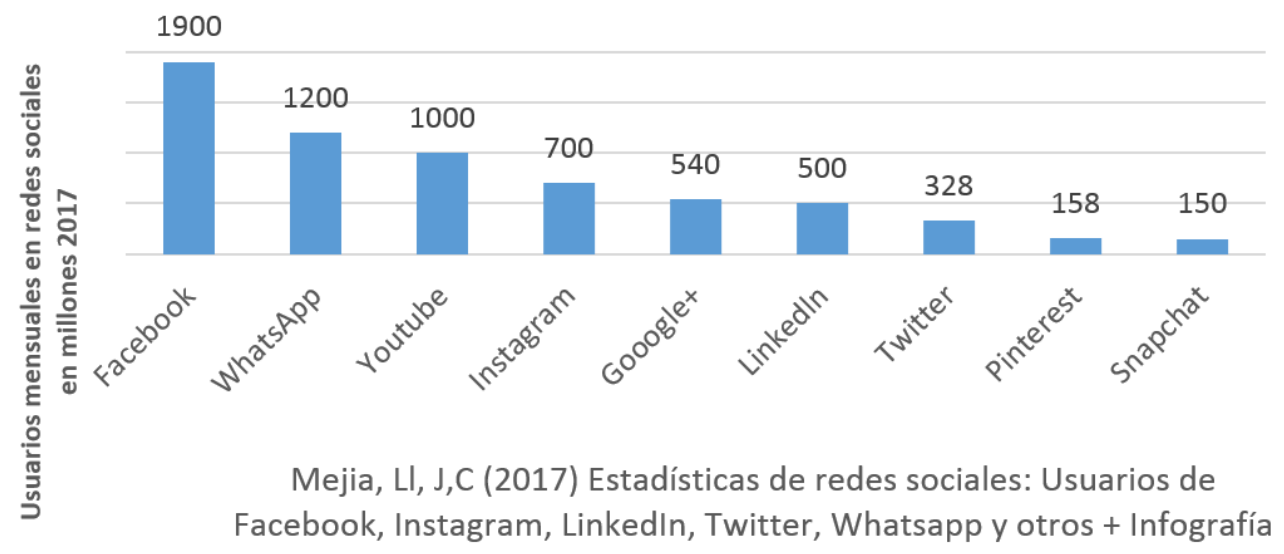

Gráfico n. 1. Uso de redes sociales 2017.

Como se puede observar, Facebook se ha mantenido entre las redes sociales más utilizadas desde su inicio, esto debido a que genera relaciones dinámicas, se realizan actualizaciones permanentemente, se puede monitorear la actividad de las cuentas, además de administrar quien puede ver las publicaciones que se emiten en ella, la información del usuario y sus contactos.

Al respecto Rebeca Valenzuela (2013), menciona que "las redes sociales forman parte de lo que se conoce como tecnologías web 2.0, y es por ello que tienen un gran potencial en la educación, ya que impulsan estudiantes activos e involucrados en su aprendizaje"; no obstante, para reconocer en las redes sociales un atributo educativo, es necesario explorar y definir cuáles son los procesos pedagógicos, los roles del docente y el alumno y la forma en que éstos se configuran en el contexto de las redes sociales.

Martín Barbero (2006) y Burbules y Callister (2001) en Pérez (2012), sugieren reorganizar la enseñanza pensando en los nuevos rasgos 
de reproducción de los saberes, como son la hipertextualidad, la interactividad, la conectividad y la colectividad. Por ello es que el proceso de adaptación de la "vieja escuela", se torna compleja, dado que requiere trasladar los procesos pedagógicos presenciales al espacio virtual. En tanto los estudiantes jóvenes conviven en dicho entorno con familiaridad, los administradores y docentes se viven en el proceso de adaptación a las tecnologías.

La era digital plantea retos acelerados que ponen en "jaque" a las instituciones educativas, siendo el desarrollo o ejecución de las competencias tecnológicas docentes, el desafío que no termina de asumirse. Al respecto Díaz Barriga (2010) en el Congreso Iberoamericano de Educación, celebrado en Argentina en el 2010, realizó una disertación en la que habló de las TIC como "elemento de trascendencia que ha transformado la vida de millones, pero también se ha reconocido que su impacto en la educación dista de sus potencialidades", aludiendo que en Latinoamérica existe un rezago en el acceso a las TIC y señala que, "entre las prioridades a atender para promover usos innovadores de las TIC en las escuelas, se encuentra el tema del desarrollo y perfeccionamiento continuo de las competencias tecnológicas y didácticas del profesorado".

Elemore y City (2011) en Pérez (2012) señalan que las escuelas, tal y como las conocemos en la actualidad, desaparecerán gradualmente, y serán remplazadas por redes sociales organizadas en torno a objetivos, propósitos de los aprendices y sus familias. Lo que ya comienza a gestarse de manera espontánea y natural, como respuesta a la convivencia de los alumnos con la tecnología, en algunas escuelas que ven a las redes sociales como opción para la comunicación con los estudiantes.

En el uso de redes sociales las ventajas, pueden representar riesgos en su uso, en el internet todo se relaciona o puede relacionarse con todo y todo está disponible al mismo tiempo, el riesgo es la dispersión y asumir información que no siempre es confiable. El internet es una red de redes interconectadas, sin centros, ni jerarquías, que controlen y filtren el intercambio. Internet puede estar anticipando una nueva forma de pensar basada más en los procesos que en los productos, en la necesidad imperiosa de sinterizar la vasta y diversa morfología actual de la información (Biltón en Pérez, 2012).

Ya se dijo que la interacción social ha dejado de ser exclusivamente "cara a cara" que las tecnologías se encuentran presentes en más espacios de convivencia social, lo que hace necesario e imposible de evitar, la incursión de la educación en medios tecnológicos y aunque el dotar a las instituciones educativas de tecnología no es sinónimo de éxito académico o calidad educativa, los administradores de la educación se preocupan en dotar de tecnología a las escuelas y los educadores tratan de dar uso a ellas.

\section{Evolución de las redes sociales}

En la literatura existen diversas descripciones que hacen referencia al surgimiento de las redes sociales mediadas por la tecnología y su incorporación al contexto educativo, no obstante, dada la trayectoria y el reconocimiento en el ámbito académico y educativo, en el estudio de las redes sociales, a 
continuación, se hace alusión a lo que Castels, Coll y Monereo rescatan sobre la historia de las redes sociales en contextos educativos.

Por su parte Castells (2001) en Coll (2008) se ha referido al Internet como un nuevo y complejo espacio global para la acción social y, por extensión, para el aprendizaje y la acción educativa. En este sentido, Valenzuela (2013:9) refiere que la incursión de las tecnologías en la educación "está relacionada con la capacidad comunicativa que brindan las tecnologías Web 2.0; su característica preponderante que es el rol activo del usuario y; que los estudiantes se encuentran involucrados y conocen muy bien diversas herramientas de redes sociales, por lo cual, el extender la educación a este ámbito es una manera de penetrar en espacios que les son conocidos a los alumnos."

El contexto en el uso de las tecnologías que vivimos hoy en día obedece a un proceso histórico en el que el hombre ha buscado incesantemente la socialización con otros y la búsqueda del conocimiento. Coll y Monerero (2008) presentan una síntesis de diversos autores a partir del cual construyen los "hitos" de la evolución de las TIC en relación al proceso de socialización y aprendizaje. En el primer hito los autores aluden a la etapa del habla y gestualidad del hombre primitivo; el segundo que hace mención del desarrollo de técnicas alimenticias, de construcción y vestimenta; en el tercero en el que describen la llegada de los sistemas de comunicación como el telégrafo, luego la radio y la televisión; para cerrar con la llegada de la Sociedad de la Información (SI), que representa un estadio más de desarrollo del ser humano, caracterizado, por la capacidad de sus miembros para obtener y compartir información de manera instantánea.
Por su parte Castells (2001), relata la historia del internet en seis lecciones, que pueden resumirse en las siguientes premisas:

1. "El internet se desarrolla a partir de la interacción entre la ciencia, la investigación universitaria fundamental, los programas de investigación militar en Estados Unidos y la contracultura radical libertaria”, el autor señala que el internet surge como un programa militar pero nunca hubo aplicación.

2. El mundo de la empresa no fue en absoluto la fuente de internet, muestra de ello es que imperios de la comunicación como ATT no vio en el internet rentabilidad.

3. El internet se desarrolla a partir de una arquitectura informática abierta y de libre acceso desde el principio; dado que el internet es abierto los productores de tecnología fueron los usuarios, tal es el caso de las redes sociales, construidas por cibernautas, más que por los norteamericanos o empresarios.

4. El internet se desarrolla desde el principio a partir de una red internacional de científicos y técnicos que comparten y desarrollan tecnologías en forma de cooperación", "internet se autogestiona, de forma informal, por una serie de personalidades que se ocupan del desarrollo de Internet sin que el Gobierno se meta demasiado con ellos, finalmente refiere que "el acceso a los códigos de Internet, el acceso a los códigos del software que gobierna Internet, es, ha sido y sigue siendo abierto". 
Hoy en día, gracias al internet y al uso de las redes sociales en educación, la formación escolarizada ha extendido sus posibilidades para formar en nuevos entornos, ya sea a través de recursos creados para ello, como el uso de plataformas como moddle o el aprovechar la popularidad de las redes sociales como el Facebook, el sistema educativo tiene de frente amplias posibilidades de generar estrategias pedagógico-didácticas que le permitan dar respuesta a las nuevas generaciones y aprovechar los recursos que las TIC le ofrecen.

Existen otros investigadores como Ponce (2012) quien realiza un recorrido histórico para hablar de la evolución de las redes sociales, la autora refiere algunos momentos clave, como el envío del primer correo electrónico en 1971 entre dos ordenadores situados uno al lado del otro, la creación de sitios web con el lanzamiento de GeoCities en 1994, el surgimiento de la red social Classmates, para contactar antiguos compañeros de estudio; la creación de Instant Messenger en 1997 por parte de AOL, hasta la llegada de Facebook en 2003 y; el servicio de alojamiento y socialización de videos en Youtube en el 2005.

Lo anterior se torna complejo frente a la mirada del investigador, dado que el objeto de estudio cambia y evoluciona de manera permanente.

\section{Noción de redes sociales}

La Web 2.0 trajo consigo la evolución de las redes sociales, que fueron de la presencialidad a la virtualidad, las cuales han permitido establecer lazos de socialización y comunicación en todo el mundo, además del libre acceso a la información.
Hoy en día podríamos comunicarnos casi en cualquier rincón del mundo con personas que ubicadas a miles de kilómetros en tiempo real o de forma asíncrona.

Investigadores como Rebeca Valenzuela (2013), argumenta que "las redes sociales forman parte de lo que se conoce como tecnologías web 2.0, y es por ello que tienen un gran potencial en la educación, ya que impulsan estudiantes activos e involucrados en su aprendizaje"; no obstante, para que el uso de las redes sociales como herramienta educativa tengan el efecto esperado es necesario reconfigurar los procesos pedagógicos, los roles del docente y el alumno, dado que las redes sociales en sí mismas no tienen la posibilidad de educar, por tanto es indispensable que exista una intencionalidad en el uso de las redes sociales por parte de quien las utiliza y que detone situaciones que puedan dar como resultado el aprendizaje.

El término de red social comenzó a utilizarse en el campo de las ciencias sociales como la sociología y la antropología para describir a un conglomerado de personas que interactúan. Entre los diferentes conceptos que existen sobre el término, se puede mencionar Sanz Menéndez (2003), quien refiere que una red social es un conjunto de relaciones sociales o interpersonales que ligan individuos $u$ organizaciones en grupos y que es al interior de estos grupos que se establecen reglas de convivencia, modos de interacción, aprendizajes, etcétera.

Como puede leerse en el párrafo anterior, las redes son formas muy antiguas de la actividad humana, pero actualmente, a la luz de la tecnología, han cobrado un nuevo sentido epistemológico, teórico, conceptual 
y de abordaje metodológico, al convertirse primero en redes sociales mediadas por la tecnología y luego, en redes sociales de información impulsadas por Internet. "Hoy en día, las redes tienen extraordinarias ventajas como herramientas organizativas debido a su flexibilidad y adaptabilidad. Características fundamentales para sobrevivir y prosperar en un entormo que cambia a toda velocidad" (Castells, 2001).

Por otro lado, Félix Requena Santos de la Universidad de Málaga (1989), define red social como una "serie de vínculos entre un conjunto definido de actores sociales, donde los vínculos, tienen la propiedad de proporcionar interpretaciones de la conducta social de los actores implicados en la red".

Por lo anterior se entiende por red social al grupo de personas que se aglutinan en un espacio de interacción, persiguiendo intereses u objetivos comunes, que pueden descifrarse a partir de los vínculos que establece y las personas con quienes se vincula; se dice que es una red social mediada por la tecnología porque la socialización ocurre en un espacio virtual. Para el caso de esta investigación se considera la red social de Facebook.

\section{Educación y redes sociales: Un nuevo desafío en los procesos de enseñanza y aprendizaje}

Las redes sociales y la tecnología están modificando la forma en que la escuela y los estudiantes se están comunicando dentro y fuera de las instituciones educativas, ya que en la era digital se generan recursos tecnológicos para que los estudiantes se apropien de otras realidades sociales y educativas, tal es el caso de los cursos virtuales que operan en diferentes plataformas educativas, con el respaldo institucional o la comunicación que se establece con otras personas de forma virtual.

En este escenario las redes sociales mediadas por el docente, pueden ampliar los modos de apropiación y empoderamiento de los estudiantes a través de nuevos espacios para socializar información y por ende las formas de aprender en congruencia con las tendencias de la sociedad de la información.

Existen algunas experiencias que consideran las redes sociales como herramientas didácticas alrededor del mundo, tal es el caso de la Universidad de Málaga (Gómez 2012) y Santiago de Compostela (Tuñez 2012) en España, quienes reconocen las cualidades de las redes sociales en la comunicación con los estudiantes. En México existen referentes en la Universidad Pedagógica Nacional (Isaak, 2014), en donde se abordaron las bondades del Facebook en cuanto a comunicación con los estudiantes. Además de las anteriores se pueden encontrar en redes sociales cientos de casos no documentados, donde los profesores cuentan con un grupo dentro de sus cuentas de Facebook u otras redes, en las que se comunican permanentemente con sus estudiantes para fortalecer sus procesos didácticos.

En consonancia con los nuevos esquemas de aprendizaje en el marco de la era digital, se debe reconocer que existe un reto que deben asumir las instituciones educativas y quienes se dedican a la tarea de enseñar, ya que es una realidad que el avance y crecimiento desmedido de la tecnología educativa les implique su dominio y 
uso en la cotidianidad didáctica, tal es el caso de las redes sociales que ya son de uso común para una gran cantidad de personas dentro de las comunidades escolares.

Como ya se mencionó con antelación, la red social más utilizada en El Salvador y el mundo es Facebook, por ello se tiene la hipótesis de que puede ser utilizada con fines didácticos y como un canal para llevar el trabajo del aula a un contexto externo a ella y aprovechar la versatilidad de ésta para compartir materiales de aprendizaje o incluso para adentrarse en el conocimiento específico de algunos contenidos en particular o mejor aun cuando las redes sociales sirven para realizar foros o se constituyen un imprescindible espacio de comunicación educativa.

Otra de las bondades que se observan en las redes sociales en el ámbito educativo es que puedan constituirse como herramientas formativas que sirvan para incentivar el uso adecuado de buenas metodologías de enseñanza-aprendizaje y con ello transmitir el conocimiento en forma ágil y oportuna a través de la divulgación de los aportes científicos y académicos vigentes.

Además de lo anterior, se promueve el aprendizaje autogestivo y permanente, lo cual impide la creación de un aprendizaje estático o un conocimiento muerto ya que parte de la intención educativa de las redes sociales aplicadas en la enseñanza es que estas puedan ayudar al docente a trabajar en una democratización del conocimiento y una real difusión y socialización del mismo.

La variabilidad de las redes sociales en ámbitos formativos, contribuye a la idea de que, en un mismo ambiente de enseñanza pueda ser posible encontrar modos y medios de compatibilizar una diversidad de estilos de aprendizaje, esto se debe a que la misma dinámica de trabajo en redes sociales en donde todos se comunican con todos, permita incluir modos fáciles y prácticos de realizar trabajos académicos en forma colaborativa $y$ cooperativa basadas en la horizontalidad junto con didácticas convencionales.

Otro de los aspectos por los cuales debería de considerarse a las redes sociales elementos de gran valor en el proceso de enseñanza-aprendizaje, es el denominado uso de las tecnologías, ya que ello motiva al docente a reconocer que el uso de internet durante el desarrollo de la clase con sus estudiantes genera que estos hagan uso de un gran número de fuentes de información y de otros recursos tecnológicos ya que, el uso de redes sociales impulsa el fomento de competencias y nuevas destrezas que probablemente con el aprendizaje tradicional no puedan lograrse, ello se debe a que las redes sociales facilitan una gestión de comunicación más efectiva debido al gran número de alumnos y profesores con los que se puede estar en permanente contacto para buscar una mejor productividad en el aprendizaje del estudiantado.

Al respecto, Rodríguez (2016), afirma que "el uso de las redes sociales en el desarrollo de la clase, permite que, en el interior y exterior del aula, se pueda incrementar la colaboración del estudiante, docente y otros estudiantes". Esto es posible gracias a la intervención que hace el docente a través de instrucciones precisas del trabajo que habrá de realizarse por parte de los estudiantes, como la búsqueda de tareas o trabajos grupales. Agrega que las redes sociales 
son útiles al momento "de que surgen dudas sobre alguna asignación de tarea, los estudiantes pueden mediante un simple mensaje, exteriorizar su duda a fin de que alguien pueda responderles". También se pueden compartir recursos al usar las redes sociales (...). Asimismo, el profesor genera mayor participación de los estudiantes compartiéndoles enlaces en una determinada cuenta que tengan en común”(Rodríguez, 2016).

No obstante, estos resultados no pueden darse por sí solos, ya que las redes sociales no tienen estas bondades en sí mismas, por lo que se requiere direccionar su uso a través de la mediación docente. Prueba de ello es el estudio realizado por Gómez (et al) publicado en el 2014, en el que dan cuenta del uso que dan estudiantes universitarios a las redes sociales, encontrando en primer lugar el envío de mensajes, la socialización de fotografías, uso del chat, ver noticias o videos, estar al pendiente de eventos, reproducir música, hacer notas, conocer enlaces de contenido, crear grupos de interés y jugar.

Aunque en la investigación anterior, queda claro que el uso académico no está dentro de los usos que estos estudiantes dan al Facebook, dentro de este mismo estudio se encontró que el $59.9 \%$ de los alumnos valoraban como positiva la posibilidad de crear un grupo de la asignatura dentro de alguna red social.

Con lo anterior se busca justificar la manipulación (en el buen sentido de la palabra) por parte del docente, de la red social, a través de instrucciones precisas a los alumnos, que permitan establecer un andamiaje, para el uso del Facebook como Entorno Personal de Aprendizaje a partir del uso de algunos contenidos temáticos.
Siendo congruentes con las proyecciones tecnológicas y el lento crecimiento que tienen los sistemas educativos en América Latina, no cuesta entender que el rol de las redes sociales en educación tienen que vincularse a un proyecto pedagógico con secuencia didáctica en el cual se enmarque el trabajo metodológico del docente, teniendo claro que cada experiencia del estudiante con la tecnología no es innovadora por sí misma, por lo que lo que realmente transforma la percepción de innovación es el hecho de hacer que las redes sociales en educación puedan ayudar a los estudiantes a apropiarse de los diferentes saberes que se promueven en una asignatura.

Ahora bien ¿por qué las redes sociales se vuelven más atractivas para los estudiantes y por qué puede ser una herramienta para el aprendizaje? Para dar respuesta, se comenzará por citar a Parra (2010), quien en su artículo "Las redes sociales de Internet: también dentro de los hábitos de los estudiantes universitarios", hace mención de algunas particularidades que vuelven atractivas las redes sociales para la población joven cuya ocupación es la formación universitaria.

En primera instancia Parra menciona como un momento trascendental el momento en que el internet trascendió la computadora para utilizarse a través de dispositivos móviles como el celular; otro atractivo de las redes sociales es que permite establecer relaciones sociales con diferentes personas en cualquier parte del mundo con afinidades, intereses y objetivos comunes; además las redes sociales son una opción de entretenimiento.

Como se puede observar en la investigación anterior, el potencial que vuelve atractivo a las redes sociales es su enorme capacidad de fomentar 
el aspecto comunicacional entre los estudiantes, lo cual pudiera ser aprovechado para extenderlo con sus profesores, sus amigos y compañeros de otros cursos, lo que probablemente puede potenciar en positivo la dinámica educativa de los ambientes de trabajo escolares, del cual se desprende en forma directa el gran aporte de las redes sociales al sistema educativo.

Otro atractivo para el usuario de redes sociales es la apertura que tiene para emitir un estilo de expresión, logrando con ello fomentar más y mejores relaciones sociales con sus pares, su atractivo esta aunado a la libertad y apertura de modificar en forma particular su ambiente al incorporar, a sus espacios interactivos, las fotografías, videos, libros, música, imágenes y demás elementos que contribuyan positivamente a su espacio virtual, lo cual puede trasladarse al ambiente educativo.

De lo anterior se puede decir que las redes sociales son útiles herramientas que se han ido posicionando en el ambiente escolar cuando se piensa en mejorar los procesos educativos, ya que éstas poseen infinidad de beneficios que contribuyen en gran medida a la construcción de saberes.

En alusión a la concordancia de lógica educativa del presente artículo, la fundamentación del mismo se desarrollara teniendo como contexto Facebook, ya que es por antonomasia la red social por excelencia debido a su fácil manejo, destacando que no es ni ha sido diseñada para labores educativas, pero en el uso práctico de muchos docentes, ha demostrado que es una herramienta que puede contribuir a la mejora continua de la labor docente, ya que al ser de uso virtual puede estar al alcance de cualquier persona con acceso a internet, sea por medio de una Laptop, un Smartphone o Tablet.

Por otro lado, el Facebook garantiza la fácil interacción entre la red y quienes la conforman, contribuye a que los docentes puedan crear gran variedad de estrategias de abordaje y seguimiento a sus clases; un claro ejemplo pueden ser los foros virtuales dentro de las redes, espacios de interacción digital en el cual los profesores determinan las reglas de participación, en donde los educandos pueden resolver las dudas que tengan sobre cierto tema en cualquier momento. También pueden crear un grupo privado donde se incluyan a todos los estudiantes a los que el docente imparte clase y así poder administrar un solo espacio educativo donde se fomente el aprendizaje colectivo. Lo mejor de Facebook es que puedes seguir en tiempo real la participación de los demás, ya que las notificaciones llegan directamente al correo electrónico, o al dispositivo móvil.

\section{Las redes socialesy su potencial enla construcción de entornos personales de aprendizaje}

En la actualidad los entornos de aprendizaje han ido de la familia, el grupo de pares, la escuela, el maestro y los libros impresos, al uso de la web 2.0 y dentro de ella, las plataformas virtuales que permiten el aprendizaje a través de entornos digitales y otros espacios no formales como las redes sociales, las cuales abren el abanico pedagógico a un nuevo contexto enmarcado en la era digital, que ofrece posibilidades de acceso a la información de forma ilimitada.

De acuerdo a Castañeda y Adell (2013), “el entorno natural de nuestras interacciones se ha 
expandido a la red de información que hemos tejido globalmente en las últimas décadas", generando un "cambio de paradigma de trabajo, desde una pedagogía que cree en el aprendizaje por exposición de la información, a una que pone el énfasis en aprender haciendo $y$, sobre todo, en aprender a aprender para poder seguir aprendiendo a lo largo de toda la vida".

Lo anterior abre un campo de investigación centrado en la construcción de aprendizaje, no solo en el entorno natural (escuela, familia, grupo de pares, docentes, etc.), si no en el entorno digital, que incluye a las redes sociales mediadas por la tecnología, en este contexto surge el concepto de Entorno Personal de Aprendizaje.

Un entorno personal de aprendizaje es el conjunto de herramientas, fuentes de información, conexiones y actividades que cada persona utiliza de forma asidua para aprender, en ellos se consideran los siguientes elementos: leer para acceder a la información, hacer, que implica reflexionar haciendo, compartir en la red personal de aprendizaje (Adell y Castañeda, 2010).

Los entornos de aprendizaje han trascendido el espacio escolar, áulico y la instrucción del profesor para trasladarse a otro, la web, la cual ofrece posibilidades de acceso a la información en cualquier momento y espacio, lo único que se requiere es la conexión a internet.

En este sentido, Sue Waters citado por Adell (2013) mencionó que "el uso de herramientas web tales como blogs, wikis, Twitter, Facebook, para crear conexiones con otras personas que extienden nuestro aprendizaje, incrementan nuestra reflexión mientras nos permiten aprender juntos como parte de una comunidad global"; para el trabajo colaborativo ya no es indispensable la sincronía y presencialidad, todo gracias a las bondades de la tecnología que hace posible el trabajo a distancia en diferentes espacios temporales.

Waters plantea a las redes sociales como un espacio para el desarrollo del aprendizaje colaborativo, en el que las interacciones entre los sujetos son una realidad dentro de la virtualidad, por lo tanto, las redes sociales se reconocen como un espacio de socialización que de forma espontánea genera aprendizaje.

Frente a los entornos de aprendizaje existen dos posturas encontradas tanto para su exploración como para la aplicación; una que considera que los entornos de aprendizaje tienen una independencia de las instituciones y que los estudiantes aprenden más por sí mismos, sostienen que para hablar de entornos de aprendizaje es menester una construcción natural no instruida por parte del aprendiz.

La otra postura denominada entornos personales de aprendizaje mediadas por la Institución (iPLE por sus siglas en inglés), consideran que los estudiantes no poseen de manera innata las habilidades para vivirse en su entorno en un continuo aprendizaje y generar sus PLE y que, por tanto, es necesario dotarles (desde la institución) de habilidades para desarrollar sus propios PLE, en esta perspectiva los PLE son una nueva metodología educativa.

Como se mencionó en un inicio, una de las posturas de algunos investigadores respecto a los PLE, es la que defiende la idea sobre que 
los estudiantes requieren apoyo por parte de los educadores para que los primeros, hagan un buen uso de las TIC como PLE, pues no poseen las habilidades suficientes para ello.

Los PLE potenciados institucionalmente, como los denominan algunos autores (Casquero, Portillo, Ovelar, Romo, y Benito, 2014), son espacios digitales pre-configurados que ofrecen una base para que el estudiante pueda organizar, en función de sus intereses y objetivos, su propia red de recursos, aplicaciones y herramientas para aprender, y su propia red de personas con quienes involucrarse en un momento dado en actividades de aprendizaje.

Según Coll (2008), los estudiantes no aprovechan, o aprovechan poco, las posibilidades que les ofrecen los PLE potenciados institucionalmente, las causas de este hecho hay que buscarlas en la falta de habilidades tecnológicas de los estudiantes, la falta de tiempo para configurar y personalizar el entorno o su falta de interés. Para otros, las causas se encuentran en la carencia de ayudas y apoyos por parte del profesorado.

En este mismo sentido, Ruiz Palmero, Sánchez García y Gómez García (2013), de la Universidad de Málaga, realizaron un estudio con el objetivo de conocer el papel que los entornos personales de aprendizaje (PLE) juegan en la formación del alumnado de la Facultad de Ciencias de la Educación de la Universidad de Málaga, los investigadores, llegaron a la conclusión que los estudiantes no usan de manera eficaz los medios con los que cuentan y que los que lo hacen no asocian el empleo de los PLE con usos académicos.
Con el antecedente de investigaciones fundamentadas de que los estudiantes no utilizan los recursos como PLE, puede justificarse que desde las instituciones hagan intervenciones didácticas que permitan colocar un andamio metodológico a los estudiantes para que encuentren en los espacios tecnológicos su propio entorno de aprendizaje.

Para efectos de la investigación se retomará la perspectiva de los iPLE, que sugieren Coll y Engel, se retomará el concepto de diseño tecnopedagógico que sugiere Coll, a través del cual se establecerá una ruta a seguir por parte de los alumnos, este permitirá establecer un andamiaje para que los alumnos recreen su propio entorno personal de aprendizaje para que luego, en otro contexto tengan ese aprendizaje y puedan trasladar la experiencia vivida a su cotidianidad.

Esto es posible, ya que como se ha visto, un entorno que se crea por una o varias personas para aprender usando las tecnologías en red, por tanto, el docente es una de esas personas que puede incidir positivamente en el desarrollo de habilidades para el uso de las TIC como un entorno de aprendizaje.

Académicos de la talla de Víctor Hugo Pacheco Castro del Instituto Salvadoreño para el Desarrollo Educativo, señala que las redes sociales en ámbitos educativos deberán estar debidamente estructuradas en algunos elementos que son los verdaderos responsables de hacer de las redes sociales un componente efectivo durante el desarrollo del hecho educativo (Pacheco, 2013).

Pacheco Castro va más allá al argumentar que cuando las personas escuchan hablar del termino 
redes sociales, la primera reacción es pensar en Facebook o Twitter. Pero para él es sumamente relevante destacar que estas no son las únicas, ya que en la actualidad existen una gran cantidad de redes sociales hechas específicamente al ámbito educativo por lo que ofrecen grandes posibilidades en la mejora de la educación.

En consonancia con lo anterior, Pacheco sitúa tres grandes componentes de las redes sociales que se describen a continuación:

1. La comunicación en las redes sociales: elemento indispensable para establecer un contacto directo con muchas personas en diferentes latitudes y con la bonanza de poder interactuar sin necesidad de un espacio físico.

2. Asistencia o apoyo: este elemento permite recurrir a las redes sociales como un soporte del trabajo colaborativo en donde además de favorecer las múltiples interacciones, se va construyendo una sólida fuente de información con todos los aportes proporcionados por los estudiantes.

3. Conjunción de ideas: En este apartado se destaca la noción de que por medio de las redes sociales se puede llegar a más personas aumentando el número de estudiantes con quienes se puede crear espacios de interacción educativa, creando un grupo más amplio del que se podría tener en una comunidad presencial.

En resumen, el interés del componente formativo de las redes sociales ayuda a que la comunicación entre el docente y su grupo de estudiantes sea directa y sencilla, logrando que acciones como la publicación de un simple mensaje en el "muro" pueda cobrar tal importancia debido a la dimensión y magnitud del contenido informativo que quiere transmitirse por lo que la red social tiene un efecto directo en la mejora de la comunicación personal estudiante-profesor.

\section{Los objetivos formativos en el uso de redes sociales}

Cuando se habla de objetivos formativos en el uso de redes sociales en contextos educativos, nos referimos a los indicadores o metas que los docentes y conglomerados estudiantiles se proponen cumplir con el uso de las redes sociales en educación. El uso de estas supone la creación de una serie de mecanismos didácticos que ayuden a concretar el perfil deseado en una asignatura o plan de estudios.

Es importante reconocer para el uso de redes sociales en contextos formativos, se deben tener objetivos de aprendizaje claros y definidos de cómo y por qué de las actividades y contenidos de enseñanza, ya que dichos objetivos se convierten en los criterios de funcionamiento que responden a las preguntas fundamentales acerca de la planeación, realización y evaluación del proceso enseñanza-aprendizaje bajo el uso condicionado de la herramienta tecnológica.

Lasntein Marshall Jones de la Universidad de Ontario señala que:

La aplicación correcta de un proceso de enseñanza y aprendizaje bajo un enfoque utilitario en el que las redes sociales son los protagonistas tecnológicos, ayuda a determinar que la importancia de usar 
objetivos formativos en dichos procesos se vuelve relevante porque con su uso e implementación se facilita la comunicación entre lo que quiere lograr el docente con sus estudiantes, ante lo cual se deben considerar los siguientes aspectos:

A) El uso de objetivos formativos en el desarrollo de contenidos de clase usando redes sociales ayuda a que todos los involucrados sepan a donde quieren llegar con el estudio y abordaje de la temática.

B) El uso de objetivos formativos durante el proceso de aprendizaje bajo el esquema de redes sociales permite que el estudiante pueda identificar el logro del esfuerzo que el mismo pone para aprender.

C) El uso de objetivos formativos durante el proceso de aprendizaje bajo el esquema de redes sociales propicia un mejor aprovechamiento del tiempo de la clase.

D) El uso de objetivos formativos durante el proceso de aprendizaje bajo el esquema de redes sociales favorece el trabajo de grupo, puesto que los propósitos con los que se desarrollan las clases son comunes para todos, además ayuda a la creación de un clima estimulante para el aprendizaje permitiendo además desarrollar evaluaciones justas $y$ objetivas. (Jones, 2014)

Una clara interpretación del planteamiento de Jones, permite entender que una definición de objetivos de aprendizaje en ambientes educativos donde el recurso tecnológico es uno de los protagonistas principales es importante porque constituye la base para que toda la comunidad educativa ubique el camino que toma el aprendizaje, es decir reconocer hacia dónde vamos en educación usando redes sociales. Además, permite al docente mejorar en su marco de eficiencia durante el abordaje del proceso de enseñanza-aprendizaje.

\section{Abordaje metodológico}

La intención del presente artículo de investigación es tener impacto en los ámbitos social y académico, además de que aporte recursos para dotar a la sociedad de herramientas para enfrentar los cambios vertiginosos a los que es sometida, sobre todo en el uso de la tecnología. Para el caso de esta investigación, el futuro deseable, viable y factible se refiere en los siguientes párrafos:

Facebook es una herramienta, que mediada por el docente puede ser efectiva para la promoción de procesos de construcción de saberes, dado que no se pueden dejar éste proceso como producto de la generación espontánea, sobre todo cuando existen objetivos curriculares definidos.

A su vez, Facebook podría ser un recurso para que los estudiantes trasladen el aprendizaje formal a otros contextos con los que contactan a través de $\mathrm{FB}$, para ello es necesario contar con un proceso instruccional que permita a los estudiantes tener claridad de cómo utilizar el contenido de la asignatura en otras áreas con las que tiene contacto a través de la red social, es decir, Facebook puede ser un puente entre el aprendizaje dentro del aula y el contexto social.

El concepto Entorno Social de Aprendizaje puede extenderse a las redes sociales con un 
método de uso aprendido por los alumnos, el cual puede ser mediado por el profesor.

Para que esto sea posible se requiere inversión de tiempo para habilitar a los estudiantes en el uso del Facebook en el contexto de la asignatura, por lo que será necesario el tener objetivos claros e instrucciones precisas que permitan tener mejores condiciones para el trabajo tanto del alumno como del profesor.

Será indispensable que dentro de la mediación el docente considere: propiciar que los estudiantes encuentren que usos pueden darle al FB para su aprendizaje, provocar el trabajo grupal y en comunidad, generar el feedback entre los alumnos y su comunidad, colocar andamios para generar interacciones constructivas en términos de aprendizaje, entre los estudiantes.

Para que lo anterior sea posible se pretende llevar a cabo un estudio de caso de corte cualitativo a través de técnicas etnográficas, donde se inducirán procesos de ayuda didáctica en el uso de redes sociales (Facebook). Los objetivos que se persiguen son:

Analizar el proceso de desarrollo de competencias para la construcción de los entornos de aprendizaje, a través de la herramienta de FB, en estudiantes universitarios, a partir de la construcción, aplicación y evaluación de una secuencia didáctica. $\mathrm{Y}$ en la intervención: Habilitar condiciones ambientales y procesos didácticos que movilicen habilidades para la construcción de Entornos Personales de Aprendizaje (PLE), en redes sociales mediadas por la tecnología, en estudiantes del nivel superior.
Para tal efecto se pretende que el uso de redes sociales en los procesos educativos genere lo siguiente:

- Habilidades para el uso de la herramienta tecnológica.

- Habilidades colaborativas.

- Habilidades de socialización.

- Persona activa que busca, crea, adapta y difunde contenidos.

- La ayuda y ajustes de la ayuda.

- Categorías de diseño instruccional

- Desarrollo de habilidades para utilizar las redes sociales como un PLE, tales como: el control y la gestión del aprendizaje, donde el estudiante fija sus objetivos y comunica a otros sus ideas.

- Organización de la información.

- Manejo de los diferentes modelos de intervención en crisis.

- Elaboración de propuestas para la solución de conflictos de otras personas.

Para ello se consideran los siguientes instrumentos de recolección de la información, con los cuales será posible hacer contacto con los datos.

- Método etnográfico: Para describir el proceso de construcción de un PLE a través de la rede social de Facebook. 
- Herramientas de triangulación: La observación, donde se analizarán, habilidades para el uso de la herramienta tecnológica, habilidades colaborativas, habilidades de socialización, persona activa que busca, crea, adapta y difunde contenidos, la ayuda y ajustes de la ayuda

- Entrevistas con alumnos, donde se explorarán percepciones respecto a las categorías de análisis.

\section{Consideraciones éticas: Las redes sociales y la privacidad del estudiante}

Una óptica moderna de ver a las redes sociales aplicadas a contextos educativos es entenderlas como estructuras sociales que se puede representar en forma de relaciones formativas en donde todos aprenden de todos, generándose con ello un gran flujo de información y conocimiento en el que la estructura social educativa se adapta perfectamente a profesores y alumnos mediante relaciones educativas representadas en clases, contenidos, cursos, tutorías, grupos de trabajo interdisciplinar, etcétera.

Las redes sociales no son controlables por parte de los docentes por lo que, en el plano educativo, la seguridad de los estudiantes es un elemento a considerar siempre. El docente como coordinador de la labor educativa llevada a cabo desde las redes sociales debe tener el total control del contenido que es apto y del que no es apto, volviéndose necesaria una adecuada preparación del profesor en el uso y manejo de redes sociales en ámbitos educativos.

Por consiguiente, la labor del docente/ investigador en ambientes virtuales hace que, en muchas ocasiones, el trabajo con estudiantes obligue a interactuar con redes cerradas para evitar la violación de la privacidad por personas ajenas al grupo de estudios. Como ya se ha dicho en numerosas ocasiones, los usos formativos de las redes pueden tener una inimaginable cantidad de usos para trabajar una o varias áreas específicas del conocimiento, lo cual permite la eficacia en el trabajo entre profesores y los estudiantes, por lo que una red social educativa puede ser un medio muy eficaz que muy probablemente, aumente la responsabilidad educativa de los propios estudiantes, esto se debe gracias a que el educando deja de ser solo un espectador para ser partícipe activo su propio proceso educativo ya que al usar la tecnología permite poder relacionarse, valorar, compartir contenidos, organizar materiales y hasta comunicar resultados evaluativos.

El tema de la privacidad en cuanto al uso y manejo de redes sociales, está íntimamente relacionado con el modo y la forma en la que el estudiante decide cómo y en qué orden acceder a la amplia cantidad de datos que encuentra en la red. No obstante, la velocidad en el aprendizaje de los usos de recursos tecnológicos no ha evolucionado con la misma rapidez que el desarrollo de las temáticas de estudio. En un amplio sentido de interpretación de lo que es y representa la privacidad en las redes sociales educativas, los profesores dejan de ser meramente comunicadores o transmisores de conocimiento para pasar a ser tutores que guían a los estudiantes mediante un proceso educativo que intenta potenciar la participación de los mismos. Viera de las Casas (2015), ha señalado que algunos de los beneficios centrados en la privacidad del estudiante al utilizar redes sociales en educación son la mejora de los ambientes de trabajo y aumenta la fluidez y sencillez en la comunicación de sus miembros. 
La importancia de cuidar la privacidad del estudiante cuando se recurre al uso de las redes sociales para la labor educativa radica en que ésta contribuye a la mejora del entornoylas condiciones de trabajo y por ende en el rendimiento académico. Para nadie es desconocido que las redes sociales tienen un enorme atractivo en el aspecto personal y de relación por parte del estudiante, por este motivo, se acierta al decir que cuanto mayor sea el número de los participantes más atracción genera en los alumnos el poder estar en contacto directo con sus profesores, sus amigos y compañeros de otros cursos a los que quizás conozcan de vista pero con los que no ha hablado nunca, creando un ambiente de trabajo favorable centrado en las redes sociales.

Una red social segura es una red social que posee el innegable valor de acercar el aprendizaje informal y el formal en donde el estudiante puede atender a las exigencias propias de su educación.

\section{Para cerrar}

En cuanto a la aportación de este apartado bibliográfico, este se encuentra dentro del ámbito pedagógico didáctico y para llevarlo a cabo se tendrá como plataforma teórica la Teoría constructivista socio cultural, inspirada en las ideas y planteamientos vigotskyanos.

Conceptualmente, será considerada la noción de Entorno Personal de Aprendizaje (PLE) que han desarrollado Adell y Castañeda ${ }^{2}$ y

2 Un Entorno Personal de Aprendizaje (PLE por sus siglas en inglés), de acuerdo a Adell (2010) es "el conjunto de materiales, aplicaciones y servicios digitales y recursos humanos que una persona utiliza para aprender en los diferentes contextos educativos y profesionales en los que participa" (Coll \& Engel, Introducción al monográfico los entornos personales de aprendizaje en contextos de educación formal., 2014). que se encuentra enmarcada en el ámbito del uso de tecnologías, como herramienta para el aprendizaje. A las ideas de Adell se suma la propuesta de Casquero, quien introduce el concepto de Entornos Personales de Aprendizaje mediados por la institución iPLE. ${ }^{3}$

Se tomará en cuenta la idea de diseño pedagógico, desde el concepto de la "diseño tecnopedagógico" de César Coll, ${ }^{4}$ a través de la cual se propone establecer un andamiaje que permita ayudar al alumno a establecer su propio entorno de aprendizaje, lo cual puede ser trasladado al uso de redes sociales mediadas por la tecnología.

Las principales aportaciones que este artículo da a la ciencia es que se tendrá una experiencia dentro de la tradición constructivista en el ambiente tecnológico en donde se trabaje con el desarrollo de Entornos Personales de Aprendizaje en redes sociales, para lo cual se utilizará la red social de Facebook, además se desarrollará una propuesta pedagógico-didáctica para ayudar a los estudiantes a generar su propio PLE en dicho medio.

Desde el punto de vista tecnológico, el desarrollo de una investigación basada en el argumento sostenido en el presente artículo, podría ser el inicio de la generación de

3 Los PLE potenciados institucionalmente, como los denominan algunos autores (Casquero, Portillo, Ovelar, Romo, y Benito, 2014), son espacios digitales pre-configurados que ofrecen una base para que el estudiante pueda organizar, en función de sus intereses y objetivos, su propia red de recursos, aplicaciones y herramientas para aprender, y su propia red de personas con quienes involucrarse en un momento dado en actividades de aprendizaje.

4 Diseño tecnopedagógico que sugiere Coll, a través del cual se establecerá una ruta a seguir por parte de los alumnos, este permitirá establecer un andamiaje para que los alumnos recreen su propio entorno personal de aprendizaje para que luego, en otro contexto tengan ese aprendizaje y puedan trasladar la experiencia vivida a su cotidianidad. 
software que permitan la socialización de información y la generación de conocimientos con las bondades y ventajas que ofrece una red social como Facebook, que forma parte de la cotidianidad de los sujetos y así abonar a otros proyectos, disciplinas y enfoques.

\section{Referencias}

Adell, J. y Castañeda, L. (2010). Los entornos personales de aprendizaje (PLEs): una nueva manera de entender el aprendizaje. En Roig, R. y Fiorucci, M. (eds). Claves para la investigación en Innovación y calidad educativas. La integración de las Tecnologías de la Información y la Comunicación y la Interculturalidad en las aulas. (19-30) Alcoy: Marfil.

Birmingam. (2017). Internet numbers and figures for the analysis. Maryland: Maryland SUN Books.

Casquero, O.; Ovelar, R.; Romo, J. y Benito, M. (2014). Entornos de aprendizaje personales, educación superior y analítica del aprendizaje: un estudio sobre los efectos de la multiplicidad de servicios en las redes personales de estudiantes universitarios. Cultura y Educación, 26, pp. 696-738. https:// doi.org/10.1080/11356405.2014.985945

Castañeda, L. y Adell, J. (Eds.). (2013). Entornos Personales de Aprendizaje: claves para el ecosistema educativo en red. Alcoy: Marfil.

Castells, M. (2001). La galaxia internet. Madrid: Editorial Arete.

Chan, M. (s/f). De las plataformas a los ambientes educativos personalizados. Recupe- rado de: http://dione.cuaed.unam.mx:3003/ nramirez/PASAPAGINAS/EDM1/files/ assets/downloads/page0219.pdf

Coll, C. y Engel, A. (2014). Introducción al monográfico los entornos personales de aprendizaje en contextos de educación formal. Cultura y Educación, 26, pp. 617-630.

Coll, C.; Engel, A.; Saz, A. y Bustos, A. (2014). Los entornos personales de aprendizaje en la educación superior: del diseño al uso. Cultura y Educación, 26, pp. 775-801.

Coll, C. y Monereo, C. (2008). Psicología de la educación virtual. Madrid: Morata.

Díaz Barriga, F. (2010). Las TIC en la educación y los retos que enfrentan los docentes. Congreso Iberoamericano de Educación. Metas 2021. Argentina.

El Economista. (5 de diciembre de 2016). Uso de redes sociales en México creció casi 14\% en 3T. Recuperado de: desde http://eleconomista. com.mx/tecnociencia/2016/12/05/usoredes-sociales-mexico-crecio- $139-3 \mathrm{t}$

Gómez, M.; Roses, S. y Farías, P. (2012). El uso académico de las redes sociales en universitarios. Comunicar, 38. https://doi. org/10.3916/C38-2012-03-04

Isaak, F. (2014). Propuesta de Estrategia Docente: El uso de Facebook como espacio para promover el trabajo colaborativo (T.C.) fuera del aula, en la licenciatura de Psicología Educativa de la Universidad Pedagógica Nacional. AMIC (Eds.). Memoria 
electrónica del XXVI Encuentro Nacional de AMIC. San Luis Potosí, México: AMIC.

Jones, G. (2014). An introduction to higher education y Canada. In K. M. Joshi an Saee Paivandi (eds.) Higher education across nations (vol. 1, pp. 1-38). Delhi: B. R. Publishing.

México digital. (9 de febrero de 2017). Transformación educativa. Recuperado de: https://www.gob.mx/mexicodigital/ articulos/transformacion-educativa-95278

Pacheco, C. (2013). Redes sociales y ámbitos educativos en educación básica. San Salvador: Ediciones e Impresos Marsall

Parra, E. (2010). Las redes sociales de Internet: también dentro de los hábitos de los estudiantes universitarios. Anagramas Rumbos y Sentidos de la Comunicación. 9, pp. 107-116. Recuperado de: <http://www.redalyc.org/ articulo.oa? $\mathrm{id}=491549024008>$

Pérez, G. A. (2012). Educarse en la era digital. Madrid: Morata.

Ponce, I. (2012). Redes Sociales. Historia de las redes sociales. Observatorio tecnológico. Gobierno de España. Ministerio de Educación, Cultura y Deporte. Recuperado de: http://recursostic. educacion.es/observatorio/web/es/internet/
Requena, S. F. (1989). El concepto de red social. Reis: Revista española de investigaciones sociológicas. 48. Recuperado de: http://www. jstor.org/stable/40183465 ?seq=1\#page_ scan_tab_contents

Ruiz, P.; Sánchez, R. y Gómez, G. (2013). Entornos personales de aprendizaje: estado de la situación en la facultad de Ciencias de la Educación de la Universidad de Málaga. Revista de Medios y Educación 42, pp. 171181. http://hdl.handle.net/10486/661357

Sanz, M. L. (2003). Análisis de redes sociales: o cómo representar las estructuras sociales subyacentes. Apuntes de Ciencia y Tecnología, 7, pp. 20-29. Recuperado de: http://ipp.csic.es/ sites/default/files/content/workpaper/2003/ dt-0307.pdf

Túñez López, M. y Sixto, J. (2012). las redes sociales como entorno docente: análisis del uso de Facebook en la docencia universitaria Pixel-Bit. Revista de Medios y Educación, 41, pp. 77-92. Recuperado de: http://www. redalyc.org/articulo.oa? $\mathrm{id}=36828247006$

Woods, P. (1987). La escuela por dentro. La etnografía de la investigación educativa. Barcelona: Paidós. 